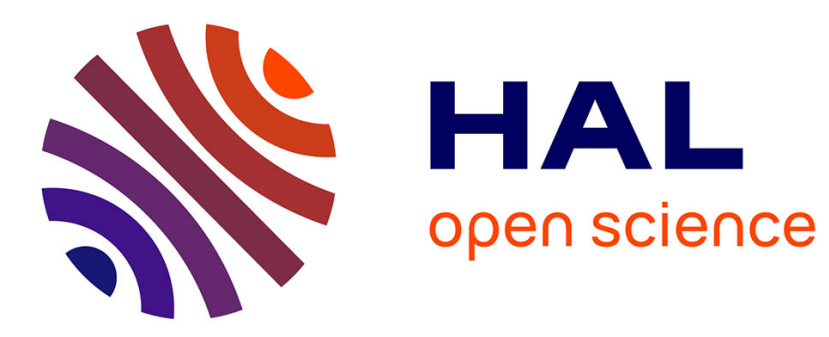

\title{
A Toy Model to Investigate Stability of AI-Based Dynamical Systems
}

\author{
Blanka Balogh, D. Saint-martin, A. Ribes
}

\section{To cite this version:}

Blanka Balogh, D. Saint-martin, A. Ribes. A Toy Model to Investigate Stability of AI-Based Dynamical Systems. Geophysical Research Letters, 2021, 48 (8), 10.1029/2020GL092133 . hal-03482715

\section{HAL Id: hal-03482715 \\ https://hal.science/hal-03482715}

Submitted on 16 Dec 2021

HAL is a multi-disciplinary open access archive for the deposit and dissemination of scientific research documents, whether they are published or not. The documents may come from teaching and research institutions in France or abroad, or from public or private research centers.
L'archive ouverte pluridisciplinaire HAL, est destinée au dépôt et à la diffusion de documents scientifiques de niveau recherche, publiés ou non, émanant des établissements d'enseignement et de recherche français ou étrangers, des laboratoires publics ou privés. 


\section{Geophysical Research Letters}

\author{
RESEARCH LETTER \\ 10.1029/2020GL092133 \\ Key Points: \\ - A higher dimensional extension of \\ the Lorenz' 63 model is introduced to \\ investigate instability issues \\ - When trained on a single orbit, \\ feedforward neural networks can \\ generate unstable trajectories \\ - Instability issues can be solved \\ by using a well-designed learning \\ sample
}

Supporting Information:

Supporting Information may be found in the online version of this article.

Correspondence to:

B. Balogh,

blanka.balogh@meteo.fr

Citation:

Balogh, B., Saint-Martin, D., \& Ribes, A. (2021). A toy model to investigate stability of AI-based dynamical systems. Geophysical Research Letters, 48, e2020GL092133. https://doi. org/10.1029/2020GL092133

Received 17 DEC 2020 Accepted 30 MAR 2021

(c) 2021. American Geophysical Union. All Rights Reserved.

\section{A Toy Model to Investigate Stability of AI-Based Dynamical Systems}

\author{
B. Balogh${ }^{1}$ (D) D. Saint-Martin ${ }^{1}$ (D), and A. Ribes ${ }^{1}$ (D) \\ ${ }^{1}$ CNRM, Université de Toulouse, Météo-France, CNRS, Toulouse, France
}

\begin{abstract}
The development of atmospheric parameterizations based on neural networks is often hampered by numerical instability issues. Previous attempts to replicate these issues in a toy model have proven ineffective. We introduce a new toy model for atmospheric dynamics, which consists in an extension of the Lorenz'63 model to a higher dimension. While feedforward neural networks trained on a single orbit can easily reproduce the dynamics of the Lorenz' 63 model, they fail to reproduce the dynamics of the new toy model, leading to unstable trajectories. Instabilities become more frequent as the dimension of the new model increases, but are found to occur even in very low dimension. Training the feedforward neural network on a different learning sample, based on Latin Hypercube Sampling, solves the instability issue. Our results suggest that the design of the learning sample can significantly influence the stability of dynamical systems driven by neural networks.
\end{abstract}

Plain Language Summary Typical atmospheric models use parameterizations to represent processes occurring at a scale smaller than the model resolution (e.g., clouds, convection, etc.). Recently, artificial intelligence, and in particular neural networks, have been described as being of high interest to improve parameterizations. Nevertheless, first attempts to develop parameterizations based on machine learning have often led to numerical instability issues. Toy models commonly used in atmospheric research are not complex enough to allow the study of these instabilities, as they are easily learned by neural networks. Here, we introduce a new toy model for atmospheric dynamics. Neural networks fail to reproduce the dynamics of this new toy model, resulting in unstable trajectories. However, training the neural network on a specifically designed learning sample, which explores the phase space comprehensively, solves the instability issues. Our results suggest that the design of the learning sample can significantly influence the stability of dynamical systems driven by neural networks. These results are expected to have practical impacts on the development of neural-network based parameterizations, and, thus, on the improvement of atmospheric models.

\section{Introduction}

Many efforts have been made over the last few years to take advantage of artificial intelligence (AI) in atmospheric and climate modeling. One line of research has sought to develop new, data-driven parameterization schemes to replace part of an atmospheric model (e.g., Brenowitz \& Bretherton, 2018; Gentine et al., 2018; O’Gorman \& Dwyer, 2018); learning a ML-based parameterization scheme means learning to predict time derivatives for sub-grid scale atmospheric processes (e.g., turbulence, and convection). Even though they promise numerically affordable yet accurate physics for low resolution atmospheric models (e.g., climate models), current state-of-the-art AI parameterizations are still biased and, more importantly, they face numerical instability issues. As reported by Rasp (2020), neural networks (NNs) are often numerically unstable, when coupled to the large-scale atmospheric fluid mechanics solver (e.g., Brenowitz \& Bretherton, 2019; Rasp et al., 2018). Parameterizations based on random forests (RF) have been reported to be stable (Yuval \& O'Gorman, 2020). But, when compared offline, NN-based parameterizations seem to outperform RF-based parameterizations (Brenowitz, Henn, et al., 2020).

The lack of stability in NN-based parameterizations can be interpreted as a result of breaking physical laws. Some authors propose techniques to ensure compliance with these laws (e.g., Beucler et al., 2019). Brenowitz, Beucler, et al. (2020) shows that instabilities are related to the linearized behavior of NNs when coupled to idealized wave dynamics. To fix numerical instabilities, Rasp (2020) proposes a coupled online learning 
method where a NN model, which was first trained offline, is continuously corrected according to online prediction errors. This concept is illustrated using the Lorenz'96 model (Lorenz, 1996).

To better understand the origin of stability issues, resorting to a toy model can be useful. Lorenz'96 (Lorenz, 1996, hereafter, L96) and Lorenz'63 (Lorenz, 1963, hereafter, L63) models often serve as toy models for atmospheric modeling, drawing simplified versions of the atmospheric flow. They have been extensively used as a test bed for assessing the use of data-driven methods in atmospheric models. L63 model has been accurately learned by feedforward neural networks (Scher \& Messori, 2019) or reservoir networks (Pathak et al., 2017). L96 dynamics has also been accurately learned by feedforward NNs, recurrent NNs or generative adversarial networks (Chattopadhyay et al., 2020; Dueben \& Bauer, 2018; Gagne et al., 2020), without reporting stability issues. Both L63 and L96 toy models appear to be insufficiently complex to study numerical instabilities encountered with more complex systems.

Currently, no toy model for atmospheric dynamics is currently available to investigate such instability issues encountered when developing parameterization schemes. The first aim of this paper is to introduce a simple, chaotic system challenging feedforward NNs by exhibiting numerical instabilities. Even though the two-level L96 model has often been used to address parameterization problems (i.e., learning only part of the dynamical system), this system will be based on the L63 model. This new toy model is aimed at replicating stability issues, when learning to predict time derivatives of the dynamical system with NNs. The second objective is to propose a possible method to ensure stability of the NN-generated trajectories, which in our case involves using a specific learning sample. Both objectives can be seen as steps towards solving numerical instabilities-an issue which remains challenging to develop suitable NN-based parameterizations for atmospheric models.

Section 2 describes the Lorenz'63 model and illustrates that this model is not complex enough to challenge NNs. In Section 3, we introduce the embedded Lorenz'63 model, and show that learning of this simple model with NNs leads to unstable trajectories. In Section 4, a method to address these instability issues is proposed. Finally, conclusions and discussions will be drawn in Section 5.

\section{Learning the Lorenz'63 Model}

By expanding the set of partial differential equations of Rayleigh-Bénard convection into Fourier series and then truncating, Lorenz (1963) derives a finite system of ordinary differential equations, given by:

$$
\begin{aligned}
& \dot{x}_{1}=\sigma\left(x_{2}-x_{1}\right), \\
& \dot{x}_{2}=x_{1}\left(\rho-x_{3}\right)-x_{2}, \\
& \dot{x}_{3}=x_{1} x_{2}-\beta x_{3} .
\end{aligned}
$$

The evolution of the state variable $\mathbf{x}=\left(x_{1}, x_{2}, x_{3}\right)$ is governed by the set of parameters $(\sigma, \rho, \beta)$. L63 admits a chaotic solution for some values of these parameters, in particular $\sigma=10, \rho=28$ and $\beta=8 / 3$. We use these values in the following. Although computationally cheap, L63 is able to mimic some properties of the atmospheric flow. Hence, it is a suitable toy model to perform proof-of-concept experiments.

Recently, the L63 model has been used as a test bed for assessing the use of data-driven methods in atmospheric models. The system of Equation 1 can be formally rewritten:

$$
\dot{\mathbf{x}}(t)=f(\mathbf{x}(t)) .
$$

Data-driven methods provide an approximate function, $\hat{f}$, to replace the "true" function $f$, and thus they replace the initial dynamical system by:

$$
\dot{\mathbf{x}}(t)=\hat{f}(\mathbf{x}(t)) .
$$

In this way, the dynamical system is learned through learning its derivative. Some authors have also proposed to directly learn (and predict) the future state $\mathbf{x}(t+\Delta t)$ from the current state $\mathbf{x}(t)$ (e.g., Scher \& Messori, 2019; Weyn et al., 2019, 2020). Here, we focus on learning time derivatives, as we think it is more representative of the parameterization problem. 
For the L63 model specifically, various functions $\hat{f}$ have been proposed recently, for example, using sparse linear regression (Brunton et al., 2016), feedforward neural networks (Scher \& Messori, 2019), or reservoir networks (Pathak et al., 2017). Fitting $\hat{f}$ always involves an optimization over a set of free parameters $\theta$. This optimization is done for a given loss function, and a given learning sample. In the case of dynamical systems, the learning sample is typically a time series, also called an orbit, obtained by numerical integration of the system, for example, Equation 2 . We note $\left[\mathbf{x}^{\text {orb }}\right]$ this learning sample:

$$
\left[\mathbf{x}^{\text {orb }}\right]=\left\{\left(\mathbf{x}^{n}, f\left(\mathbf{x}^{n}\right)\right)\right\}_{n=1 . . N},
$$

where $\mathbf{x}^{n}$ denotes the state of the system, $\mathbf{x}\left(t_{n}\right)$, at time $t_{n}$. To learn the L63 model, we use one single orbit as a learning sample. This orbit is obtained by integrating Equation 1 with a time-step of $\Delta t=0.05$ using a fourth-order Runge-Kutta time stepping scheme. The numerical integration is performed over 500 model time units (MTU, where $1 \mathrm{MTU}=20 \Delta t$ ). Thus, the learning sample contains $N=10^{4}$ individuals. Given the fact that the learning sample is a time series, consecutive points exhibit positive auto-correlation. As a consequence, as previously done by Scher and Messori (2019), the learning algorithm is trained on the first $80 \%$ of the learning sample, while the remaining $20 \%$ are used for test or validation.

A simple, eight-layer feedforward NN is trained to derive $\hat{f}$ using the mean squared error (MSE) as the loss function1. The hidden layers are activated by Recitifed Linear Unit function (ReLU) and the output layer is activated by a linear function. Learning is performed over 50 epochs, with Keras implementation of Adam optimizer, parameterized with an initial learning rate of 0.001 . During training, the determination coefficient $R^{2}$ over the validation subset is monitored. The training loss function $\mathcal{L}$ is the mean squared error:

$$
\mathcal{L}\left(\theta,\left[\mathbf{x}^{\text {train }}\right]\right)=\frac{1}{N} \sum_{n=1}^{N}\left\|\hat{f}_{\theta}\left(\mathbf{x}^{n}\right)-f\left(\mathbf{x}^{n}\right)\right\|^{2},
$$

where $\left[\mathbf{x}^{\text {train }}\right]$ denotes the training data set, $N$ the size of the training data set, $\mathbf{x}^{n} \in\left[\mathbf{x}^{\text {train }}\right]$ for $1 \leq n \leq N$, and $\theta$ denotes the set of parameters over which the optimization is made. Weights corresponding to the highest value of $R^{2}$ score over the validation data set are saved and loaded after the training is completed. Learning is very efficient, as we get $R^{2}=0.9998$ over the validation subset.

The integration of both the L63 model (Equation 2) and its NN-approximation (Equation 3), with the same time-step of $\Delta t=0.05$, using the same fourth-order Runge-Kutta time stepping scheme and starting from the same initial condition, show a good agreement (Figure 1). In particular, the NN-based model does not manifest any unstable behavior, that is, its orbits always lay on the Lorenz "butterfly" attractor. Repeating this analysis with different initial conditions and/or different learning samples leads to the same conclusion. This result suggests that the L63 model is not challenging enough to data-driven methods and does not allow to study instability issues, as previously shown in similar studies.

L96 provides another simple framework to study data-driven methods in the context of atmospheric modeling, and is of higher dimension than L63. However, reservoir computers and neural networks have succeeded in learning this system without reporting instability issues (Pathak et al., 2017; Scher \& Messori, 2019). Hence, both L63 and L96 appears to be too simple to encounter the typical instability issues that still hamper the development of AI parameterizations.

\section{The Embedded Lorenz'63 Model}

Building a higher dimensional model on the basis of L63 has already been proposed. Musielak and Musielak (2009) added a fourth spatial variable to extend L63 equations. Champion et al. (2019) created a high-dimensional model on the basis of L63 system by using Legendre polynomials. Here, we propose a different extension of this model specifically designed to investigate instability issues.

The key idea is as follows: the standard L63 model, which is of dimension 3, is embedded into a larger space of dimension $d>3$. In the selected 3-D subspace, the chaotic dynamics of L63 is kept unchanged. Any deviation from this 3-D subspace is brought back by a restoring force. We call "embedded" L63 model (hereafter, eL63) this new simple model. A formal definition of eL63 is in two steps (see Figure 2). 
(i) Lorenz '63 orbit

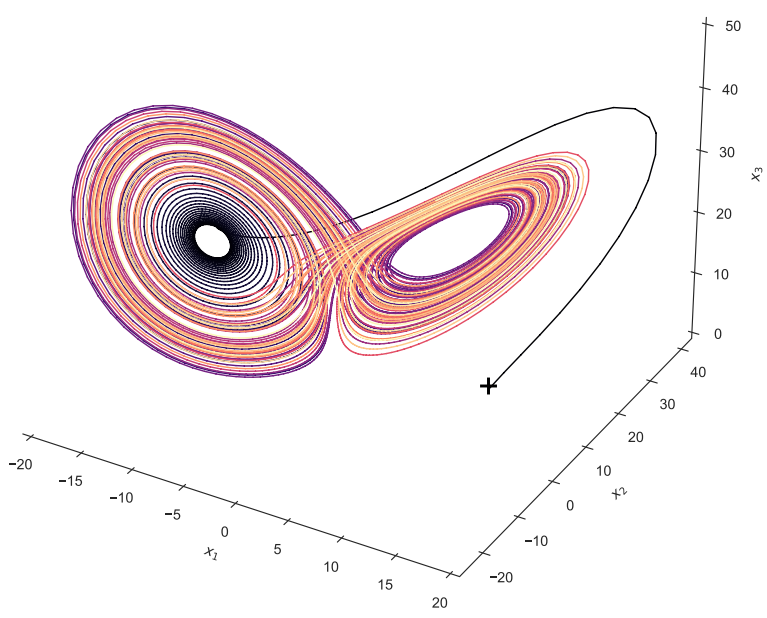

(ii) NN-based Lorenz '63 orbit

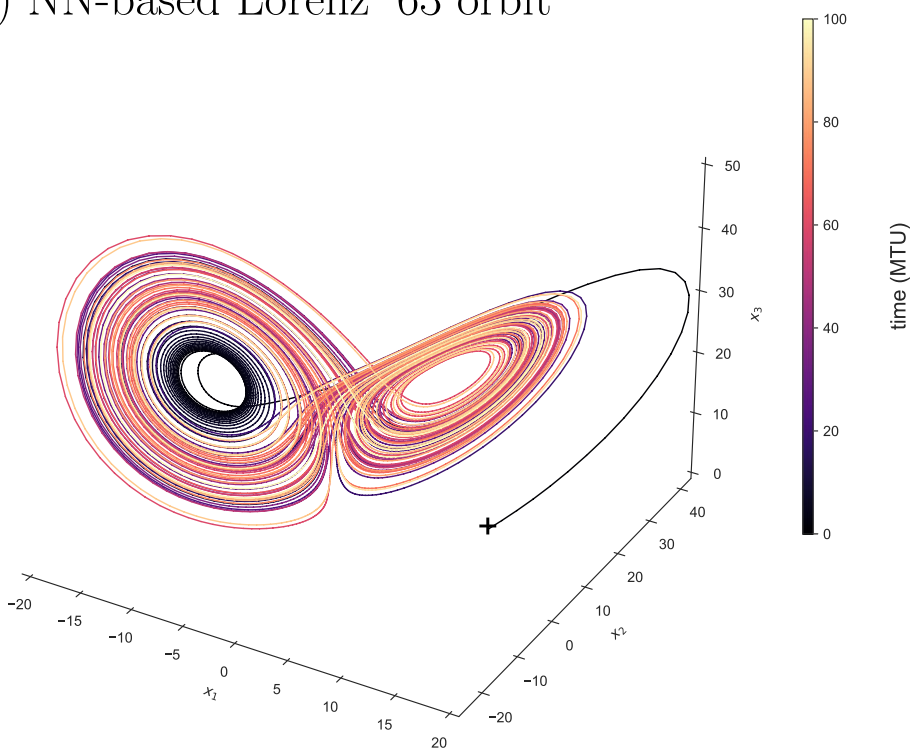

Figure 1. Examples of Lorenz'63 orbits. (i) The orbit is obtained by integration of Lorenz' 63 system of equations (see Equation 1 in the text) with $\sigma=10$, $\rho=28$, and $\beta=8 / 3$. (ii) The orbit results from the integration of neural network model $\hat{f}$ (see Equation 3 ). The numerical integration is performed over 100 model time units from initial condition $(10,15,0)$. The orbits are colored by the time variable.

(1) Embedding: we construct a vector $\mathbf{z}=\left(z_{1}, z_{2}, \ldots, z_{d}\right) \in \mathbb{R}^{d}$, and define its dynamics by:

$$
\begin{aligned}
& \dot{z}_{1}=\sigma\left(z_{2}-z_{1}\right), \\
& \dot{z}_{2}=z_{1}\left(\rho-z_{3}\right)-z_{2}, \\
& \dot{z}_{3}=z_{1} z_{2}-\beta z_{3}, \\
& \dot{z}_{j}=-\kappa z_{j}, \quad \forall j>3 .
\end{aligned}
$$

The first three equations are identical to L63, while the $d-3$ additional equations are simple restoring forces. For the sake of simplicity, the relaxation coefficient, $\kappa$, is unique. In the following, we use $\kappa=1$. We denote $\mathcal{B}_{z}$ the basis of vector $\mathbf{z}$.

(2) Random rotation: We apply a random rotation to derive the state vector of the eL63 system, $\mathbf{x}=\left(x_{1}, x_{2}, \ldots, x_{d}\right)$ :

$$
\mathbf{x}(t)=P \mathbf{z}(t),
$$

where $P \in \mathbb{R}^{d \times d}$ is the rotation matrix between $\mathcal{B}_{z}$ and $\mathcal{B}_{x}$, the basis of $\mathbf{x}$. $P$ contains the coordinates of the basis vectors of $\mathcal{B}_{x}$, seen in $\mathcal{B}_{z}$. Note that the rotation matrix $P$ does not depend on time.

$\mathbf{x}$ is the state vector of eL63, and only $\mathbf{x}$ is available to a learning procedure. The dynamics of $\mathbf{x}$ is obtained by rewriting Equations 6 and 7, such that $\dot{\mathbf{x}}(t)=f(\mathbf{x}(t))$. In this case, $f$ is the function composition of the L63 plus restoring forces and the random rotation.

A key property of eL63 is that $z_{j, j}>3$ exponentially decays towards zero and so $\mathbf{x}(t)$ is confined within a subspace of dimension 3. This subspace can be interpreted as resulting, e.g., from physical but unknown constraints. The difficulty for a data-driven method to fully capture the dynamics of eL63 comes from the fact that any orbit is very thin-almost all points are located in a subspace of dimension 3. If such an orbit is used as a learning sample, any deviation from this specific subspace in a predicted trajectory can lead to an out-of-sample issue.

We now consider the problem of learning eL63, in the same way as done for L63 in the previous section. A learning sample $\left[\mathbf{x}^{\text {orb }}\right]$ is built by integrating eL63 equations over 500 MTUs, with a timestep of $\Delta t=0.05$ 
(i) Embedding : $\mathbb{R}^{3} \mapsto \mathbb{R}^{d}$

$$
\left(z_{1}(t), z_{2}(t), z_{3}(t)\right) \mapsto \mathbf{Z}(t)=\left(z_{1}(t), z_{2}(t), z_{3}(t), \ldots, z_{d}(t)\right)
$$
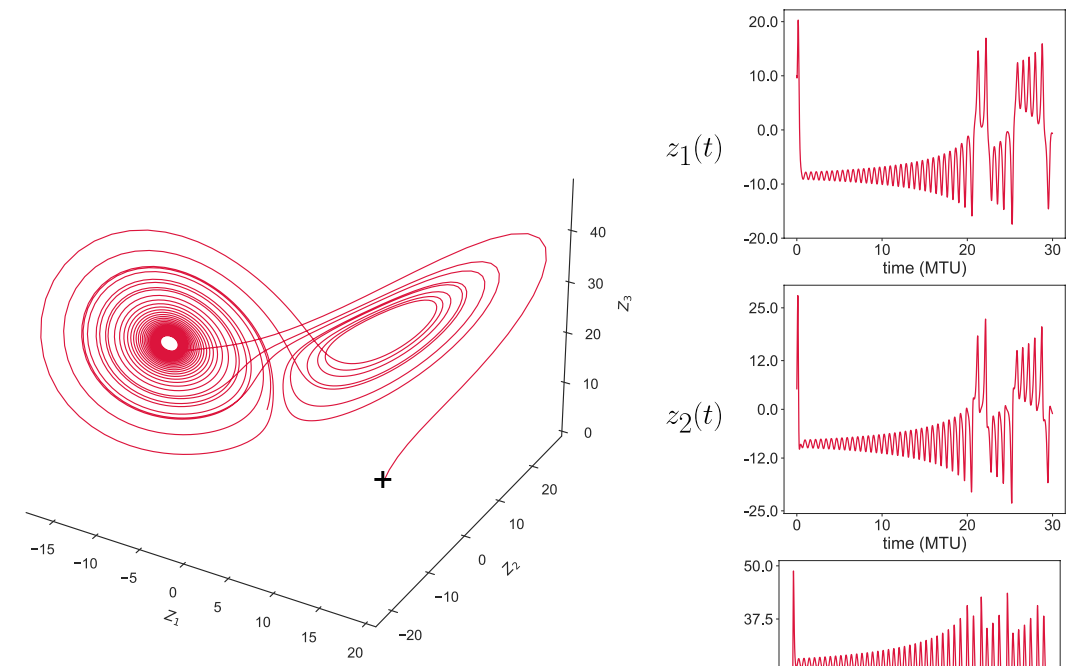

$z_{3}(t)$

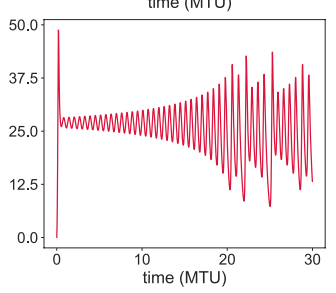

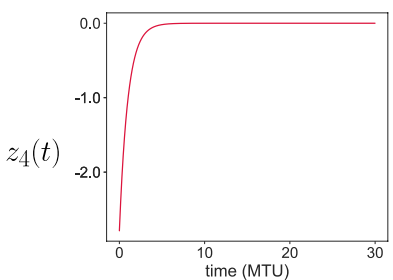

$\left(z_{5}(t), \ldots, z_{d-1}(t)\right)$

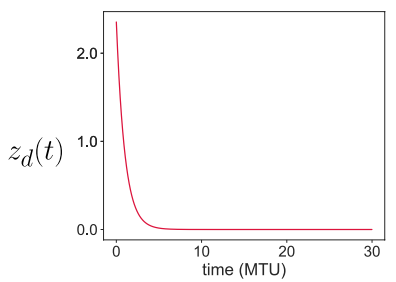

(ii) Random rotation : $\mathbb{R}^{d} \mapsto \mathbb{R}^{d}$

$$
\mathbf{X}(t)=\left(x_{1}(t), x_{2}(t), x_{3}(t), \ldots, x_{d}(t)\right)=P \mathbf{Z}(t), P \in \mathbb{R}^{d \times d}
$$
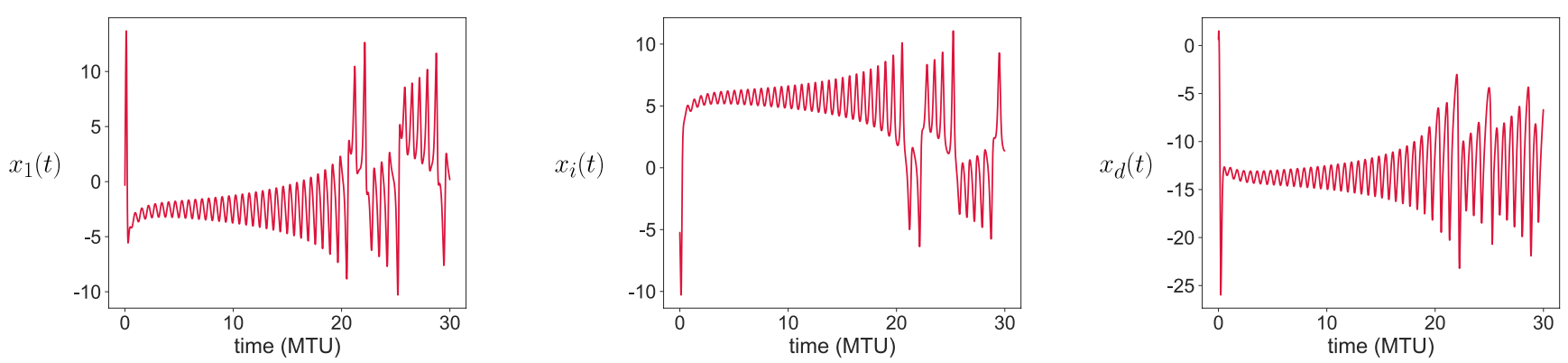

Figure 2. The embedded Lorenz'63 model (see text for details). (i) Representation of an eL63 orbit in $\mathcal{B}_{z}$ basis over 30 MTU: (left) tri-dimensional representation of $\left(z_{1}(t), z_{2}(t), z_{3}(t)\right.$ ), (middle) time series of $z_{1}(t), z_{2}(t)$, and $z_{3}(t)$, and (right) time series of $z_{4}(t)$ and $z_{d}(t)$. (ii) Representation of the same eL63 orbit in $\mathcal{B}_{x}$ basis. Time series of $x_{1}(t), x_{i}(t)$, and $x_{d}(t)$ are shown. MTU, model time units.

using a fourth-order Runge-Kutta scheme. The initial condition is sampled from an eL63 orbit, which is equivalent to removing the model spin-up from the learning data set, ensuring that $z_{j, j}>3 \approx 0$. Therefore, the learning data set contains $N=10^{4}$ individuals. The learning sample being a time series, consecutive points are positively auto-correlated. Hence, learning is performed over the first $80 \%$ of this sample, while the last $20 \%$ are saved for validation. Both the input and the target variables are then normalized so that their mean value equals 0 and their standard deviation equals 1 . A simple feedforward $\mathrm{NN}$ is trained to best approximate $f$, in the same configuration than described in Section 2 (e.g., eight hidden layers, fit over 50 epochs, 


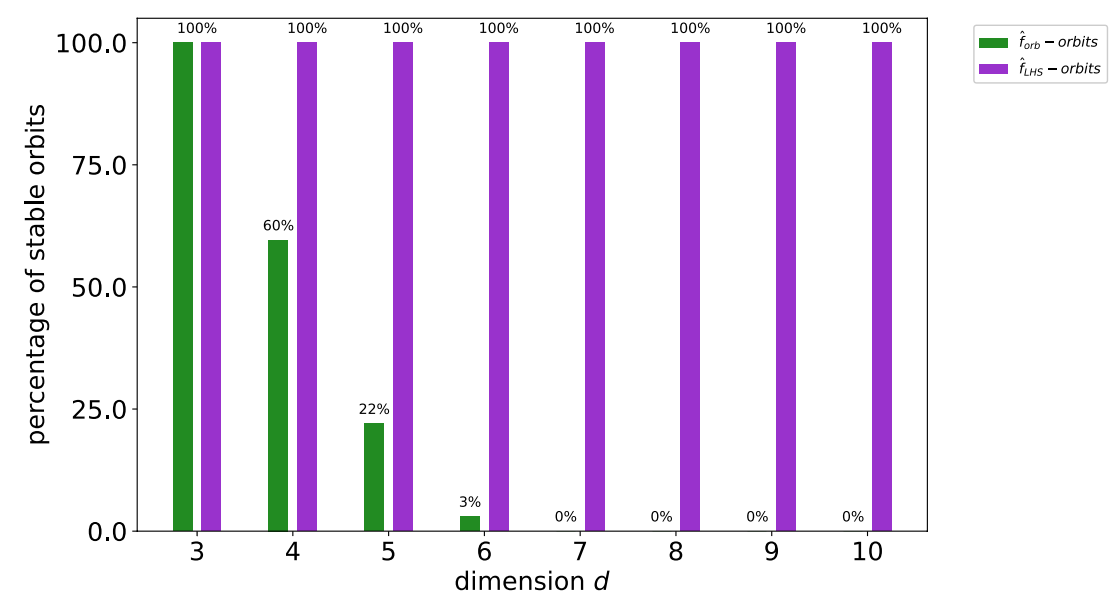

Figure 3. Percentage of stable orbits generated with $\hat{f}$ when trained with orbital $\left(\hat{f}_{\text {orb }}\right.$, green $)$ or LHS ( $\hat{f}_{\text {LHS }}$, purple $)$ learning samples, as a function of embedding dimension $d$. Initial conditions are randomly sampled either in an eL63 orbit or in a region around the eL63 attractor. Stability is assessed with 100 different $\hat{f}$ and 30 initial conditions for $d \in\{3,4,5,6,7,8,9,10\}$, using the stability criterion defined in Equation 9. LHS, Latin Hypercube Sampling.

minimizing MSE loss function). As the learning sample consists of a single orbit, we note $\hat{f}_{\text {orb }}$ the estimated function, that is,

$$
\hat{f}_{\text {orb }}=\hat{f}_{\theta^{*}} \text { with } \theta^{*}=\underset{\theta}{\operatorname{argmin}} \mathcal{L}\left(\theta,\left[\mathbf{x}^{\text {orb }}\right]\right) \text {. }
$$

We get $\mathrm{R}^{2}=0.9998$, an overall performance consistent with L63. Evidences suggest that the emulated trajectories correctly reproduce the dynamics of the eL63 system (Figure S5).

The resulting function $\hat{f}_{\text {orb }}$ is validated as follows. First, we take an initial condition within the original learning sample $\left[\mathbf{x}^{o r b}\right]$. Then, we generate the ANN-based trajectories starting from these points over 1,000 MTUs. This seems long enough for the model to manifest numerical instabilities. Integration of predicted tendencies is performed with the same time-step of $\Delta t=0.05$, and by using the same fourth-order Runge-Kutta time stepping scheme. Stability of the resulting orbit is assessed using a 'stability criterion' defined as follows: for each $i=1 . . d$, we compute the minimum $\left(m_{i}\right)$ and maximum $\left(M_{i}\right)$ values of $x_{i}$ over the training orbit $\left[\mathbf{x}^{\text {orb }}\right]$. A $N$-step trajectory is considered as stable if it remains within 7 times this range of values, that is,

$$
m_{i}-3\left(M_{i}-m_{i}\right) \leq x_{i}^{n} \leq M_{i}+3\left(M_{i}-m_{i}\right), \forall 1 \leq i \leq d, \forall 1 \leq n \leq N .
$$

The choice of this stability criterion is partly arbitrary, but it is motivated by its simplicity. The results presented here do not depend on the choice of the stability criterion, since unstable trajectories are typically explosive (see Figure S4).

The above described validation strategy is iterated over 100 different $\hat{f}_{\text {orb }}$, each being trained with a different rotation matrix $P$ and a corresponding learning sample $\left[\mathbf{x}^{o r b}\right]$. The resulting functions $\hat{f}_{\text {orb }}$ are then tested by generating orbits of length 1,000 MTU from 30 different initial conditions sampled randomly from their respective training orbit. As a result, stability can be assessed over 3,000 simulated orbits of length 1,000 MTU each. Figure 3 shows the percentage of stable trajectories generated with $\hat{f}_{\text {orb }}$ for different values of $d$, the dimension of eL63. Even with minimal embedding (i.e., $d=4$ ), 60\% of the NN-generated orbits are unstable. With $d \geq 7$, all generated orbits are unstable regarding the stability criterion defined by Equation 9 . The accumulation of small prediction errors gradually leads the NN-generated orbit away from the learning sample, in a region where $\hat{f}_{\text {orb }}$ is not accurate. Hence, many NN-generated orbits are unstable, proving that 
eL63 is a very simple model (as its dimension remains very low) which is able to reproduce instability issues. Lastly, we notice that the same recipe (i.e., embedding) can be applied to even simpler non-chaotic dynamical system, and leads to similarly unstable trajectories when learned by NNs. A simple circle, embedded in $\mathbb{R}^{3}$, seems sufficient to challenge the stability of the NN model (see Figures S1-S3).

\section{Stabilizing the NN-Based Embedded Lorenz'63 Model}

We now propose to illustrate a possible method to solve instability issues encountered by simple NN models in the case of the eL63 model. We generate a new learning sample by taking points away from a typical orbit, taking advantage of the fact that the value of $f$ can be sampled at any location. In this way, we better approximate the function $f$ on regions away from the eL63 attractors.

Here, we use a Latin Hypercube Sampling (LHS) (McKay, 1992) to generate a new learning sample of size $N=10^{4}$ (i.e., $N$ is kept unchanged). LHS generates an optimal near-random sample in a high-dimensional (here, dimension $d$ ) hypercube. The boundaries of the LHS are set to 1.5 times the range of an orbit $\left[\mathbf{x}^{\text {orb }}\right]$. This calculation is done for each $i=\{1, \ldots, d\}$, leading to an hypercube in the basis $\mathcal{B}_{x}$. Finally, we generate the target variables by simply applying $f$ to the selected points, and obtain a new learning sample $\left[\mathbf{x}^{\mathrm{LHS}}\right]$. The next step is to estimate $f$ from $\left[\mathbf{x}^{\mathrm{LHS}}\right]$. Consistent with the previous section, train and test datasets are obtained by randomly partitioning the learning sample in proportions of $80 \%$ and $20 \%$, respectively. The same, eight-layers deep feedforward NN is fitted to the LHS data in the same configuration than described in Section 2. We denote

$$
\hat{f}_{\mathrm{LHS}}=\hat{f}_{\theta^{*}} \text { with } \theta^{*}=\underset{\theta}{\arg \min } \mathcal{L}\left(\theta,\left[\mathbf{x}^{\mathrm{LHS}}\right]\right)
$$

the new estimate of $f$. Learning over the LHS sample is slightly less efficient than learning from a single orbit data set, as we find $R^{2}=0.9975$. This is an expected outcome, as the region covered by the learning sample is wider in the case of $\left[\mathbf{x}^{\mathrm{LHS}}\right]$, resulting in more complex variations of $f$.

Hundred $\hat{f}_{\text {LHS }}$ are trained over different learning datasets. The estimate functions then generate orbits of length 1,000 MTU from 30 initial conditions that has been randomly sampled from $\left[\mathbf{x}^{\text {orb }}\right]$. The same stability criterion (Equation 9) is applied to determine whether the $\hat{f}_{\text {LHS }}$ orbit is stable. Contrary to orbits generated with $\hat{f}_{\text {orb }}$, all orbits generated with $\hat{f}_{\text {LHS }}$ remain stable over 1,000 MTUs (see Figure 3) with $d=4, \ldots$, 10. This result suggests that stability can be a property of the learning sample-and not only of the type of learning algorithm or of intense tuning of hyperparameters. These results are consistent with those shown in Scher and Messori (2019) on L63 model: dynamics of the system have been learned unaccurately by a NN when data around one of the attractors or from one "wing" of the "butterfly" were removed from the training data set. It also shows that extending the training beyond the thin phase space region explored by a single trajectory is important to improve the long-term performance (in particular stability skill) of a NNbased dynamical model.

However, one caveat is worth mentioning. Even though the generated trajectories are stable, in some cases, they collapse onto a fixed point near the center of one of the eL63 attractors (see Figure S5). This seems to happen more frequently as the embedding dimension $d$ increases. It can suggest that the size of our learning sample (i.e., $10^{4}$ individuals) may not be sufficient when the embedding dimension increases. Further investigation of the issue revealed that increasing the size of the LHS learning sample from $10^{4}$ to $10^{5}$ individuals reduced the number of fixed points from $15 \%$ to less than $1 \%$ (see Figure S6). This finding could motivate further research on how to improve the design of the learning sample in such problems.

\section{Conclusion and Discussion}

We have developed an extended version of the Lorenz'63 model by embedding this model into higher dimension $d$. This new model is called the embedded Lorenz'63 model. Using a real trajectory (orbit) as a learning sample, simple artificial neural network can successfully learn the time derivatives $\dot{\mathbf{x}}$ of either 
L63 or eL63 models. However, unlike in L63, long trajectories generated by NNs are unstable in eL63. Instability is observed even with a minimal dimension increase, that is, $d=4$, and becomes more frequent as $d$ increases. As a result, eL63 is a first example of low-dimension toy model for atmospheric dynamics that can be used to investigate the stability of NN-generated trajectories. Introduction of this new model is important, because previous attempts to construct a toy model able to replicate instability issues have proven ineffective.

Using NNs of similar complexity but with a different learning sample, specifically designed using a latin hypercube sample, solves the instability issue. We interpret this result as follows. A typical eL63 orbit converges towards the model's attractor very quickly, leading to degeneracy-the orbit stays confined into a subspace of dimension 3. Conversely, a LHS generates points in the full eL63 space of dimension $d$, which allows the $\mathrm{NN}$ to learn the restoring force playing away from the attractor. This result is important, as it suggests that the design of the learning sample can largely influence the stability of the NN-generated trajectories. This finding also suggests that the design of the learning sample might be an important and potentially overlooked step in IA-based atmospheric modeling. As opposed to this, much literature to date has focused on improving the learning technique in order to ensure stability.

An important further question is whether or not this new toy model can provide helpful guidance for real-world problems, that is, developing full-complexity atmospheric parameterizations based on NNs. In our view, the response is unclear at this stage, as a few questions remain open. Is the real world as much degenerated as is eL63? The fact that any orbit gets confined into a low-dimension subspace can mimic unknown physical laws. There is a growing literature on this topic. Recent studies show that NNs cannot learn exact physical laws, typically inducing drifts in the generated trajectories (e.g., Greydanus et al., 2019). Various learning techniques have been proposed to account for such physical laws. However, the degeneracy could also result from a fast equilibrium in response to restoring forces, just as assumed in eL63 - an issue which could be more difficult to address, as physical knowledge is probably more difficult to incorporate in this case. So, if some degeneracy is likely to happen in the real-world, its strength is not well determined.

Could real-world application benefit from using designed learning samples rather than 'samples of opportunity' like single orbits? This technique first requires ability to calculate the value of $f$ at any given location. Additionally, using a LHS strategy as suggested above can be computationally demanding, especially if the dimension of $\mathbf{x}$ is large. In particular, we note that real atmospheric parameterizations involve a dimension $d$ much higher than those investigated with our toy model.

\section{Data Availability Statement}

Code is made available at: https://doi.org/10.5281/zenodo.4331710.

\section{Acknowledgments}

The authors acknowledge Stephan Rasp and one anonymous reviewer for their constructive and insightful comments on the previous version of this paper.

\section{References}

Beucler, T., Pritchard, M., Rasp, S., Gentine, P., Ott, J., \& Baldi, P. (2019). Enforcing analytic constraints in neural-networks emulating physical systems, Physical Review Letters. 126, 098302. https://doi.org/10.1103/PhysRevLett.126.098302

Brenowitz, N. D., Beucler, T., Pritchard, M., \& Bretherton, C. S. (2020). Interpreting and stabilizing machine-learning parametrizations of convection. Journal of the Atmospheric Sciences, 77, 4357-4375. https://doi.org/10.1175/JAS-D-20-0082.1

Brenowitz, N. D., \& Bretherton, C. S. (2018). Prognostic validation of a neural network unified physics parameterization. Geophysical Research Letters, 45(12), 6289-6298. https://doi.org/10.1029/2018GL078510

Brenowitz, N. D., \& Bretherton, C. S. (2019). Spatially extended tests of a neural network parametrization trained by coarse-graining. Journal of Advances in Modeling Earth Systems, 11(8), 2728-2744. https://doi.org/10.1029/2019MS001711

Brenowitz, N. D., Henn, B., McGibbon, J., Clark, S. K., Kwa, A., Perkins, W. A., et al. (2020). Machine learning climate model dynamics: Offline versus online performance. Physics: Atmospheric and Oceanic Physics, arXiv:2011.03081

Brunton, S. L., Proctor, J. L., \& Kutz, J. N. (2016). Discovering governing equations from data by sparse identification of nonlinear dynamical systems. Proceedings of the National Academy of Sciences of the United States of America, 113(15), 3932-3937. https://doi. org/10.1073/pnas.1517384113

Champion, K., Lusch, B., Kutz, J. N., \& Brunton, S. L. (2019). Data-driven discovery of coordinates and governing equations. Proceedings of the National Academy of Sciences of the United States of America, 116(45), 22445-22451. https://doi.org/10.1073/pnas.1906995116

Chattopadhyay, A., Hassanzadeh, P., \& Subramanian, D. (2020). Data-driven predictions of a multiscale Lorenz 96 chaotic system using machine-learning methods: Reservoir computing, artificial neural network, and long short-term memory network. Nonlinear Processes in Geophysics, 27(3), 373-389. https://doi.org/10.5194/npg-27-373-2020

Dueben, P. D., \& Bauer, P. (2018). Challenges and design choices for global weather and climate models based on machine learning. Geoscientific Model Development, 11(10), 3999-4009. https://doi.org/10.5194/gmd-11-3999-2018 
Gagne, D. J., Christensen, H. M., Subramanian, A. C., \& Monahan, A. H. (2020). Machine learning for stochastic parameterization: Generative adversarial networks in the Lorenz '96 model. Journal of Advances in Modeling Earth Systems, 12(3), e2019MS001896. https:// doi.org/10.1029/2019MS001896

Gentine, P., Pritchard, M., Rasp, S., Reinaudi, G., \& Yacalis, G. (2018). Could machine learning break the convection parameterization deadlock? Geophysical Research Letters, 45(11), 5742-5751. https://doi.org/10.1029/2018GL078202

Greydanus, S., Dzamba, M., \& Yosinski, J. (2019). Hamiltonian neural networks. Neural and Evolutionary Computing, arXiv:1906.01563

Lorenz, E. N. (1963). Deterministic nonperiodic flow. Journal of the Atmospheric Sciences, 20, 130-141. https://doi.org/10.1175/ 1520-0469(1963)020<0130:DNF $>2.0 . C O ; 2$

Lorenz, E. N. (1996). Predictability: A problem partly solved. Proc. ECMWF seminar on predictability (Vol. I pp. 1-18). Reading, United Kingdom: ECMWF.

McKay, M. D. (1992). Latin hypercube Sampling as a tool in uncertainty analysis of computer models. Proceedings of the 24th Conference on Winter Simulation, 557-564. USA: Association for Computing Machinery. https://doi.org/10.1145/167293.167637

Musielak, Z. E., \& Musielak, D. E. (2009). High-dimensional chaos in dissipative and driven dynamical systems. International Journal of Bifurcation and Chaos, 19(09), 2823-2869. https://doi.org/10.1142/S0218127409024517

O'Gorman, P. A., \& Dwyer, J. G. (2018). Using machine learning to parameterize moist convection: Potential for modeling of climate, climate change, and extreme events. Journal of Advances in Modeling Earth Systems, 10(10), 2548-2563. https://doi.org/10.1029/2018MS001351

Pathak, J., Lu, Z., Hunt, B. R., Girvan, M., \& Ott, E. (2017). Using machine learning to replicate chaotic attractors and calculate Lyapunov exponents from data. Chaos, 27(12), 121102. https://doi.org/10.1063/1.5010300

Rasp, S. (2020). Coupled online learning as a way to tackle instabilities and biases in neural network parameterizations: General algorithms and Lorenz 96 case study (v1.0). Geoscientific Model Development, 13(5), 2185-2196. https://doi.org/10.5194/gmd-13-2185-2020

Rasp, S., Pritchard, M. S., \& Gentine, P. (2018). Deep learning to represent subgrid processes in climate models. Proceedings of the National Academy of Sciences of the United States of America, 115(39), 9684-9689. https://doi.org/10.1073/pnas.1810286115

Scher, S., \& Messori, G. (2019). Generalization properties of feed-forward neural networks trained on Lorenz systems. Nonlinear Processes in Geophysics, 26(4), 381-399. https://doi.org/10.5194/npg-26-381-2019

Weyn, J. A., Durran, D. R., \& Caruana, R. (2019). Can machines learn to predict weather? using deep learning to predict gridded 500hpa geopotential height from historical weather data. Journal of Advances in Modeling Earth Systems, 11(8), 2680-2693. https://doi. org/10.1029/2019MS001705

Weyn, J. A., Durran, D. R., \& Caruana, R. (2020). Improving data-driven global weather prediction using deep convolutional neural networks on a cubed sphere. Journal of Advances in Modeling Earth Systems, 12(9). https://doi.org/10.1029/2020ms002109

Yuval, J., \& O'Gorman, P. A. (2020). Stable machine-learning parameterization of subgrid processes for climate modeling at a range of resolutions. Nature Communications, 11(1), 3295. https://doi.org/10.1038/s41467-020-17142-3 